\title{
The efficacy of mupirocin ointment and chlorhexidine body scrubs in the eradication of nasal carriage of Staphylococcus aureus among patients undergoing long-term hemodialysis
}

\author{
Chatrchai Watanakunakorn, MD, FACP, FCCP \\ Judy Brandt, RN, BSN \\ Pamela Durkin, RN, BSN \\ Sherry Santore, RN, BSN \\ Brenda Bota, BS, MBA, MT (ASCP) \\ Charlotte J. Stahl, RN, MSN, CIC \\ Youngstown and Rootstown, Ohio
}

Background: Patients undergoing long-term hemodialysis have a high prevalence of Staphylococcus aureus nasal carriage, which may lead to serious infections. Mupirocin ointment has been used intranasally to eradicate $S$. aureus carriage in health human volunteers and health care workers. Chlorhexidine, an antiseptic with excellent antistaphylococcal activity, is widely used for handwashing and skin cleansing. Methods: Anterior nares cultures were obtained from patients older than 18 years who were undergoing long-term hemodialysis. Patients with $S$. aureus nasal carriage were enrolled in the study. Axillae and groins were cultured. Patients were given mupirocin ointment intranasally twice per day for 7 days and chlorhexidine body scrubs daily for the first 3 days. Follow-up cultures were obtained from anterior nares, axillae, and groins at 1 day, and $1,4,8$ and 12 weeks after treatment.

Results: One day after completion of treatment nasal carriage was eradicated in $83 \%$ of patients (15/18). After 12 weeks, $69 \%$ of patients $(11 / 16)$ were free of nasal carriage. Conclusions: Success rates of eradication were excellent compared with those in other published reports. This simple and effective regimen had no major side effects. (AJIC AM J INFECT CONTROL 1992;20:138-41)

From the Infectious Disease Section and the Infection Control Services, St. Elizabeth Hospital Medical Center, Youngstown, and the Northeastern Ohio Universities College of Medicine, Rootstown.

Presented in part at the Eighteenth Annual Educational Conference and international Meeting of the Association for Practitioners in Infection Control, Inc., May 5-9, 1991, Nashville, Tennessee.

Reprint requests: Dr. C. Watanakunakorn, St. Elizabeth Hospital Medical Center, P.O. Box 1790, Youngstown, Ohio 44501-1790.

$17 / 47 / 31294$
Staphylococcus aureus is the most common cause of infection in patients undergoing longterm hemodialysis. ${ }^{1,2}$ S. aureus may cause serious infections in these patients, including bacteremia, endocarditis, infected arteriovenous fistulas and shunts, and vascular access site infections. $^{3-5}$ Most patients undergoing long-term hemodialysis are nasal carriers of $S$. aureus. ${ }^{6,7}$ Although one study reported that $S$. aureus on the vascular access site appeared to be acquired 
from the anterior nares, ${ }^{8}$ another study reported that $S$. aureus on the skin of vascular access site was associated with poor personal hygiene. ${ }^{9}$ It seems certain, however, that there is a close relationship between skin colonization and nasal carriage of $S$. aureus.

Over the years, eradication of $S$. aureus nasal carriage has been attempted with systemic and topical antimicrobial agents, with varying degrees of success.$^{10}$ However, nasal recolonization of $S$. aureus is not uncommonly detected on follow-up. Mupirocin, a recently available topical antimicrobial agent, is effective against $S$. aureus. ${ }^{11}$ Mupirocin has been used to eradicate $S$. aureus nasal carriage in healthy human volunteers, among the staff of a hospital during an outbreak of staphylococcal infections, and among residents of a long-term care institution. ${ }^{12-14}$ Chlorhexidine is an antiseptic with excellent antistaphylococcal activity. It is widely used for handwashing and skin cleansing.

The purpose of this study was to ascertain the efficacy of the application of mupirocin ointment to the anterior nares, in combination with bodyscrubs with chlorhexidine, in the eradication of $S$. aureus nasal carriage among patients undergoing long-term dialysis.

\section{METHODS \\ Patient population}

All patients more than 18 years of age in the long-term hemodialysis unit in our hospital were asked to participate in this study. Patients who had a history of allergy to either mupirocin or chlorhexidine were excluded. Written, informed consent was obtained after oral explanation. The research protocol was approved by the medical research committee and the institutional review board of the hospital. The nephrologists gave permission for their patients to participate in this study.

\section{Study design}

Screening of patients. After written, informed consent had been obtained, swabs from both anterior nares were obtained for culture. Patients whose anterior nares culture grew $S$. aureus were enrolled in this study. For patients whose anterior nares culture did not grow $S$. aureus at this time, an anterior nares culture was obtained 1 month later. If this repeated anterior nares culture grew $S$. aureus, the patient was then asked to enroll in the study.
Study protocol. Cultures of the axillae and groins were obtained from all patients whose anterior nares culture grew $S$. aureus. Demographic data were collected. Patients were given 14 packets of mupirocin $2 \%$ ointment in polyethylene glycol base and 14 swab applicators and taught how to apply mupirocin to the anterior nares twice daily for 7 days. One packet of mupirocin ointment and one swab applicator were to be used each time and discarded after use. Patients were also given three bottles of chlorhexidine solution (4 fluid ounces each) and instructed to apply the solution to the whole body from the neck down, especially to the axillae and groins, during daily baths for 3 days. One bottle was to be used each time.

Posttreatment cultures of the anterior nares, axillae, and groins were taken 1 day, 1 week, 4 weeks, 8 weeks, and 12 weeks after completion of treatment.

Microbiology. Swabs were streaked on mannitol salt agar and incubated at $37^{\circ} \mathrm{C}$ for 48 hours. Mannitol-positive colonies were tested for coagulase. Coagulase-positive colonies were designated as $S$. aureus. All $S$. aureus isolates were tested for susceptibility to penicillin and oxacillin by the standard disk diffusion test. ${ }^{15}$

\section{RESULTS}

Sixteen of the 52 patients originally cultured were $S$. aureus nasal carriers. The remaining 36 patients were cultured again 1 month later; six were then found to be $S$. aureus nasal carriers. Twenty-two patients were therefore enrolled in the study.

\section{Diabetes mellitus}

Eight (35\%) of the 23 patients with diabetes mellitus and 14 (48\%) of the 29 patients who did not have diabetes mellitus were $S$. aureus nasal carriers. Five (38\%) of the 13 patients who were receiving insulin therapy and three $(30 \%)$ of the 10 patients with diabetes mellitus but not receiving insulin therapy were $S$. aureus nasal carriers.

\section{Length of treatment by hemodialysis}

For the 22 patients who were $S$. aureus nasal carriers, the duration of hemodialysis treatment ranged from 2 to 82 months, with a mean of 35 months and median of 37 months. For the 30 patients who were not $S$. aureus nasal carriers, the duration of hemodialysis treatment ranged 
Table 1. Results of mupirocin ointment and chlorhexidine in the treatment of $S$. aureus carriage

\begin{tabular}{lcccccc}
\hline & & \multicolumn{3}{c}{ Posttreatment } \\
\cline { 5 - 7 } Source of specimens & Pretreatment & $\mathbf{1} \mathbf{d}^{*}$ & $\mathbf{1} \mathbf{w k}$ & $\mathbf{4}$ wkt & $\mathbf{8}$ wk & $\mathbf{1 2}$ wkt \\
\hline Anterior nares & $22 / 22$ & $3 / 18$ & $3 / 18$ & $5 / 17$ & $4 / 17$ & $5 / 16$ \\
Axillae & $4 / 22$ & $0 / 18$ & $0 / 18$ & $0 / 17$ & $0 / 17$ & $0 / 16$ \\
Groins & $8 / 22$ & $2 / 18$ & $1 / 18$ & $2 / 17$ & $2 / 17$ & $1 / 16$ \\
\hline
\end{tabular}

Values are expressed as number of positive cultures/number of patients cultured.

*Four patients did not complete treatment.

tOne patient died.

from 1 to 58 months, with a mean of 23 months and median of 22 months. The difference between the two groups is almost statistically significant ( $p=0.063, t=1.95$, two-tailed $t$ test).

\section{S. aureus carriage}

Results of cultures for $S$. aureus are detailed in Table 1. Four patients did not complete treatment; three of these four patients were enrolled during the second month. Two patients died during follow-up. One day after completion of treatment, nasal carriage had been eradicated in 15 of 18 patients $(83 \%)$. Twelve weeks after treatment, $69 \%(11 / 16)$ of the surviving patients were free of $S$. aureus nasal carriage. Four of the five patients had transient loss of nasal carriage but reacquired carrier status at the end of follow-up period. All five patients who were nasal carriers at the end of follow-up did not have diabetes mellitus. Only one patient had persistent positive groin culture but negative nasal culture.

\section{Antibiotic susceptibility}

All S. aureus isolates from pretreatment nasal cultures were susceptible to oxacillin and all except five isolates were resistant to penicillin. These five isolates were also tested for $\beta$-lactamase production; all were negative. One patient was also colonized before treatment with an oxacillin-resistant $S$. aureus in the axillae and groins. All posttreatment cultures from all sites of this patient had no $S$. aureus.

In two $(50 \%)$ of the four isolates from pretreatment axillary cultures and four (50\%) of the eight isolates from posttreatment groin cultures, antimicrobial susceptibility was different from that of the corresponding isolates from the nasal cultures of the same patient. In four patients, pretreatment cultures from all three sites were positive for $S$. aureus, but in two of these four pa- tients the antimicrobial susceptibility of isolates from the axillae and groins was different from that of the isolate from the anterior nares.

\section{DISCUSSION}

It has been shown that $S$. aureus nasal carriage in patients who are undergoing long-term hemodialysis is associated with more frequent $S$. aureus infections and that infections occur less frequently in $S$. aureus nasal carriers given periodic rifampin to eliminate the carrier state. ${ }^{7}$ However, resistance to rifampin develops fairly rapidly. ${ }^{7}$ In a recent study of oral ciprofloxacin plus rifampin in the treatment of patients with nasally carried methicillin-resistant $S$. aureus, resistance to ciprofloxacin also developed rapidly, necessitating the termination of the study. ${ }^{16}$

Mupirocin ointment has been used for 9 months nasally in patients undergoing long-term hemodialysis, resulting in the reduction of carriage and infection. ${ }^{17}$ With the long-term usage of mupirocin, however, development of resistance is a real concern. ${ }^{18}$ We decided to use shortterm therapy with mupirocin ointment applied intranasally, in conjunction with chlorhexidine body scrubs, in an attempt to eradicate $S$. aureus nasal carriage and prevent recolonization of the anterior nares from other body sites.

In this study, we found that the prevalence of $S$. aureus nasal carriage was not higher among patients with diabetes mellitus, either insulindependent diabetes or non-insulin-dependent diabetes, than among patients with no history of diabetes mellitus. This is in accordance with findings from a large, geographically based study.$^{19}$ Instead, the prevalence of $S$. aureus nasal carriage was related to the length of time the patient had been undergoing long-term hemodialysis. The patients who were $S$. aureus nasal carriers had been undergoing hemodialysis 
much longer than the ones who were not $S$. aureus carriers. This difference almost attained statistical significance ( $p=0.063$ ).

Our success rates of eradication $(83 \%$ immediately after treatment and $69 \%$ after follow-up for 12 weeks) are excellent, in comparison with other studies of patients undergoing hemodialysis that used topical and oral treatment regimens. ${ }^{10}$ In a study using oral and topical antimicrobial agents with a 12 -week follow-up period, the rates of eradication of $S$. aureus nasal carriage at 12 weeks were $44 \%$ and $25 \%$, for rifampin/bacitracin and bacitracin alone, respectively. ${ }^{7}$ With the chlorhexidine body-scrub treatment, axillae and groins were eliminated as sources of nasal recolonization. We used mupirocin $2 \%$ ointment in a polyethylene glycol base, which is approved by the Food and Drug Administration for topical use on skin surfaces. Mupirocin ointment in a soft paraffin base is currently under clinical investigation for intranasal use..$^{20}$ If the efficacy of this short regimen of mupirocin ointment and chlorhexidine body scrub is confirmed in large clinical trials, this treatment can be used on a periodic basis, perhaps every 3 months, for those patients who are $S$. aureus nasal carriers by repeated cultures.

We thank Beecham Laboratories, Bristol, Tennessee, for providing the mupirocin $2 \%$ ointment, and Stuart Pharmaceuticals, Wilmington, Delaware, for providing the chlorhexidine solution used in this study. We thank the nephrologists and staff of the hemodialysis unit for their cooperation in this study.

\section{References}

1. Mennes PA, Gilula LA, Andersen CB, Etheredge EE, Weerts C, Harter HR. Complications associated with arteriovenous fistulas in patients undergoing chronic hemodialysis. Arch Intern Med 1978;138:1117-21.

2. Kaplowitz LG, Comstock JA, Landwehr DM, Dalton HP, Mayhall CG. A prospective study of infections in hemodialysis patients: patient hygiene and other risk factors for infection. Infect Control Hosp Epidemiol 1988;9:53441.

3. Linnemann CC Jr, McKee E, Laver MC. Staphylococcal infections in a hemodialysis unit. Am J Med Sci 1980;276:67-75.

4. Nicholls A, Edward N, Catto GRD. Staphylococcal septicemia, endocarditis, and osteomyelitis in dialysis and renal transplant patients. Postgrad Med J 1980;56: 642-8.

5. Francioli P, Masur H. Complications of Staphylococcus aureus bacteremia. Occurrence in patients undergoing long-term hemodialysis. Arch Intern Med 1982;142: 1655-8.
6. Kirmani N, Tuazon CU, Murray HW, Parrish AE, Sheagren JN. Staphylococcus aureus carriage rate of patients receiving long-term hemodialysis. Arch Intern Med 1978;138:1657-9.

7. Yu VL, Goetz A, Wagener M, et al. Staphylococcus aureus nasal carriage and infection in patients on hemodialysis: efficacy of antibiotic prophylaxis. New Engl $J$ Med 1986;315:91-6.

8. Rebel MH, Van Furth R, Stevens P, Bosscher-Zonderman L, Noble WC. The flora of renal hemodialysis shunt sites. J Clin Pathol 1975;28:29-32.

9. Kaplowitz LG, Comstock JA, Landwehr DM, Dalton HP, Mayhall CG. Respective study of microbial colonization of the nose and skin and infection of the vascular access site in hemodialysis patients. J Clin Microbiol 1988;26:1257-62.

10. Chow JW, Yu VL. Staphylococcus aureus nasal carriage in hemodialysis patients: its role in infection and approaches to prophylaxis. Arch Intern Med 1989;149:125862.

11. Fuchs PC, Jones RN, Barry AL. Interpretive criteria for disk diffusion susceptibility testing of mupirocin, a topical antibiotic. J Clin Microbiol 1990;28:608-9.

12. Casewell MW, Hill RLR. Elimination of nasal carriage of Staphylococcus aureus with mupirocin ('pseudomonic acid') - a controlled trial. J Antimicrob Chemother 1986;17:365-72.

13. Hill RLR, Duckworth GJ, Casewell MW. Elimination of nasal carriage of methicillin-resistant Staphylococcus aureus with mupirocin during a hospital outbreak. J Antimicrob Chemother 1988;22:377-84.

14. Cederna JE, Terpenning MS, Ensberg M, Bradley SF, Kauffman CA. Staphylococcus aureus nasal colonization in a nursing home: eradication with mupirocin. Infect Control Hosp Epidemiol 1990;11:13-6.

15. National Committee for Clinical Laboratory Standards. Approved standards: M2-A4: performance standard for antimicrobial disk susceptibility tests. 4th ed. Villanova, Pennsylvania: National Committee for Clinical Laboratory Standards, 1990:1.

16. Peterson LR, Quick JN, Jensen B, et al. Emergence of ciprofloxacin resistance in nosocomial methicillinresistant Staphylococcus aureus isolates: resistance during ciprofloxacin plus rifampin therapy for methicillinresistant $S$. aureus colonization. Arch Intern Med 1990;150:2151-5.

17. Boelaert JR, De Smedt RA, De Baere YA, et al. The influence of calcium mupirocin nasal ointment on the incidence of Staphylococcus aureus infections in hemodialysis patients. Nephrol Dial Transplant 1989;4:278-81.

18. Cookson BD. Mupirocin resistance in staphylococci. J Antimicrob Chemother 1990;25:497-503.

19. Boyko EJ, Lipsky BA, Sandoval R, et al. NIDDM and prevalence of nasal Staphylococcus aureus colonization: San Luis Valley diabetes study. Diabetes Care 1989;12:189-92.

20. Reagan DR, Doebbeling BN, Pfaller MA, et al. Elimination of coincident Staphylococcus aureus nasal and hand carriage with intranasal application of mupirocin calcium ointment. Ann Intern Med 1991;114:101-6. 